\title{
The Fleur-de-PAP Flap for Bilateral Breast Reconstruction
}

\author{
Katharine Saussy, BA ${ }^{1} \quad$ Mark W. Stalder, MD ${ }^{1} \quad$ Stephen J. Delatte, MD ${ }^{2}$ Robert J. Allen, MD \\ Hugo St. Hilaire, DDS, MD ${ }^{1,2}$
}

${ }^{1}$ Division of Plastic and Reconstructive Surgery, Louisiana State University Health Sciences Center, New Orleans, Louisiana

2 Breast Cancer Specialists of Louisiana, Lafayette, Louisiana

J Reconstr Microsurg Open 2017;2:e1-e3.
Address for correspondence Hugo St. Hilaire, DDS, MD, Division of Plastic and Reconstructive Surgery, Louisiana State University Health Sciences Center, 1542 Tulane Avenue, Room 758A, New Orleans, LA 70112 (e-mail: hugost1@gmail.com).

\author{
Abstract \\ Keywords \\ - autologous breast \\ reconstruction \\ - profunda artery \\ perforator \\ - stacked flaps
}

The authors present the case of a 42-year-old female patient who underwent autologous breast reconstruction using a new perforator flap designed from the medial and posterior thigh. Due to a prior abdominoplasty, the patient's abdominal donor site was unavailable to reconstruct her large, ptotic breasts, so an alternate approach was undertaken. The fleur-de-PAP flap is based on the same perforators employed by the standard profunda artery perforator (PAP) flap, but simultaneously incorporates tissue from both the transverse and vertical PAP flap skin paddles. This design maximizes flap volume at a single donor site and provides a reasonable secondary option for autologous reconstruction in large-breasted patients in whom the abdomen is not available.
Perforator flaps have become accepted as an excellent option for autologous breast reconstruction. The deep inferior epigastric perforator (DIEP) flap is the first-line option for perforator-based flaps in most patients due to the available tissue volume, skin quality, and secondary aesthetic benefits. ${ }^{1,2}$ However, variability in patient anatomy and body habitus create the need for reliable secondary flap options.

While several perforator flaps have been used successfully for patients in whom the abdominal donor site is not available, the profunda artery perforator (PAP) flap has emerged in recent years as a popular choice due to its ease of dissection and favorable donor-site aesthetics. ${ }^{3-5}$ The ability to harvest the PAP flap in the lithotomy position makes it amendable to a simultaneous two-team approach-increasing operative efficiency-and the perforasome anatomy permits variation in skin paddle design-with either transverse PAP (tPAP) or vertical PAP (vPAP) flaps available-allowing a "designer" approach based on each patient's unique anatomy. ${ }^{6}$

We present the first described case of an immediate bilateral autologous breast reconstruction using the "fleurde-PAP" (FDP) flap design-incorporating tissue from both the tPAP and vPAP skin paddles on a single vascular pedicle.

\section{Clinical Report}

A 42-year-old female patient was referred to our clinic with a diagnosis of unilateral multifocal ductal carcinoma in situ, a plan for bilateral mastectomies, and a desire for autologous reconstruction. On examination the patient demonstrated large, ptotic breasts, and a lower abdominal scar from a previous abdominoplasty (-Fig. 1). Computed tomography angiography was obtained to evaluate perforator anatomy for free tissue transfer from the patient's thighs. To account for the volume of tissue required and the bilateral nature of the reconstruction, flaps were designed that incorporated both the tPAP and vPAP territories in a "fleur-de-lis" pattern.

With the patient in the lithotomy position, two perforators were marked out along the vertical axis of each flap, and the transverse limbs were extended from the centrally positioned vertical skin paddle to complete the markings ( $\mathbf{F i g}$. 2). The dissection began using an anterior approach, and was carried through the subcutaneous tissue and muscular fascia of the gracilis and adductor magnus muscles until the perforators were visualized. The vascular pedicle was divided at its origin to obtain sufficient length and caliber, and the harvest of the received

August 16, 2016 accepted after revision

September 8, 2016

published online

October 19, 2016
DOI http://dx.doi.org/

10.1055/s-0036-1593752. ISSN 2377-0813.
Copyright $\odot 2017$ by Thieme Medical Publishers, Inc., 333 Seventh Avenue, New York, NY 10001, USA. Tel: +1(212) 584-4662.
License terms

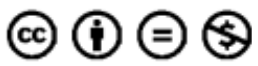




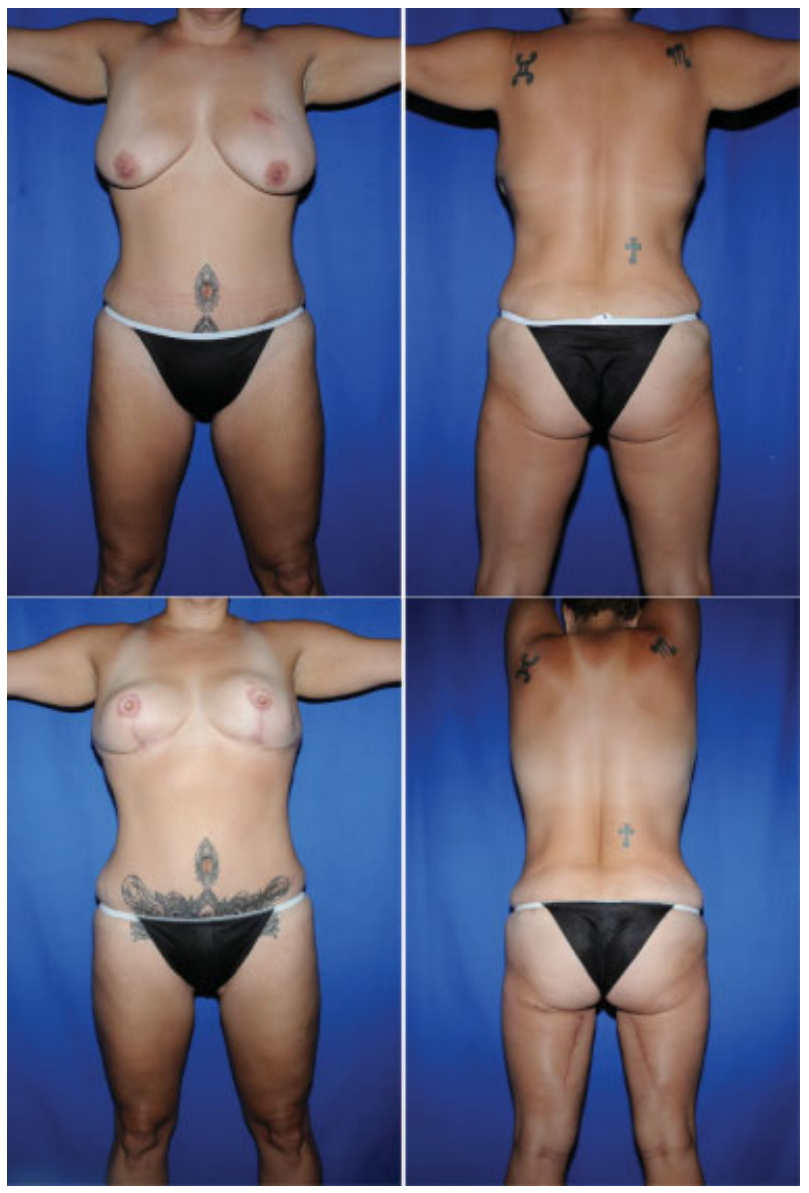

Fig. 1 Preoperative appearance of a 42-year-old female patient with unilateral multifocal ductal carcinoma in situ (upper left and right) that underwent bilateral mastectomies and immediate autologous breast reconstruction using a fleur-de-lis modification of the profunda artery perforator flap. After 6 months of the initial operation the patient underwent a second stage procedure that included bilateral fat grafting to improve contour and volume of the reconstructed breasts (lower left). No donor-site revisions were undertaken as the patient was satisfied with the postoperative appearance of her lower extremities (lower right).

posterior flap was completed using a tailor-tacking technique to avoid undue tension during the closure.

The mastectomy specimens weighed 410 and $435 \mathrm{~g}$, the flaps weighed 360 and $380 \mathrm{~g}$, respectively, and these were transferred to the chest wall for microvascular anastomosis using the internal mammary system. Inset was completed using an inverted T-position, with the lateral limbs folded posteriorly to create a three-dimensional teardrop with greater projection at the inferior pole of the breast. The patient was discharged home on postoperative day 4 . Her postoperative course was complicated by a surgical-site infection at the left breast, which responded to antibiotics, local debridement, and wound care.

A second stage procedure was completed 6 months later to finalize the reconstruction. At that time the patient underwent bilateral fat grafting with $150 \mathrm{~mL}$ of fat to each breast, and had bilateral nipple reconstruction. There were no com- plications, and the patient is pleased with the reconstructive results (-Fig. $\mathbf{1}$ ).

\section{Discussion}

As screening techniques for breast cancer have improved, the mastectomy rate has increased accordingly over recent years. ${ }^{7}$ Concurrently, outcomes in autologous breast reconstruction have improved dramatically over that time with regard to both aesthetics and morbidity. ${ }^{2,8}$ The number of reliable perforator flap options available for breast reconstruction has also increased over this period of time, allowing for greater surgical flexibility and creativity. 3,4

The DIEP flap remains the perforator flap of choice in most patients. When the abdominal donor site is not available, or contains insufficient donor tissue for an adequate reconstruction, alternative approaches must be considered. ${ }^{1,2}$ Often this correlates to the use of stacked flaps, which can be an excellent option to achieve adequate volume and projection in the reconstructed breast, while maintaining the benefits of vascularized tissue. ${ }^{2,4,5}$ Bipedicled and stacked DIEP flaps are frequently used to good effect for unilateral reconstructions. ${ }^{2}$ However, bilateral stacked perforator flap reconstructions require the use of a second donor site, typically from the gluteal or thigh areas, and are challenging cases for even the most experienced microsurgeons. ${ }^{2,5}$

The PAP flap has emerged as a popular option for stacked unilateral cases and in conjunction with the DIEP for stacked bilateral reconstructions. The PAP offers improved donor-site aesthetics and pedicle length, and an easier dissection relative to other secondary options such as the superior gluteal artery perforator or inferior gluteal artery perforator flaps. ${ }^{3,5,6,9}$ For the patient presented here undergoing bilateral autologous reconstruction of large breasts, stacked DIEP/PAP flaps would have been the preferred technique, but with the abdominal donor site unavailable the fleur-de-lis modification of the PAP flap offered an acceptable secondary option.

The FDP flap incorporates tissue from both the tPAP and vPAP skin paddles into a single flap-similar to the design of an extended medial thigh lift-maximizing volume, and employing a pseudostacked flap design on a single vascular pedicle. The design is comparable in many ways to the fleurde-lis modification of the transverse upper gracilis (TUG), which is also considered a reasonable secondary option for autologous breast reconstruction. ${ }^{10}$ However, the TUG flap relies on the vascular pedicle to the gracilis muscle, which offers considerably less length than the PAP, and necessitates sacrifice of the muscle, resulting in increased donor-site morbidity relative to the perforator-based $\mathrm{FDP}^{3,9}$

\section{Conclusion}

The FDP flap, incorporating both the tPAP and vPAP flap skin paddles into a single flap, provides yet another perforatorbased alternative for autologous breast reconstruction. This flap offers satisfactory tissue volume for moderate-sized breasts, and a reliable vascular dissection in an area already familiar to most microsurgeons. 

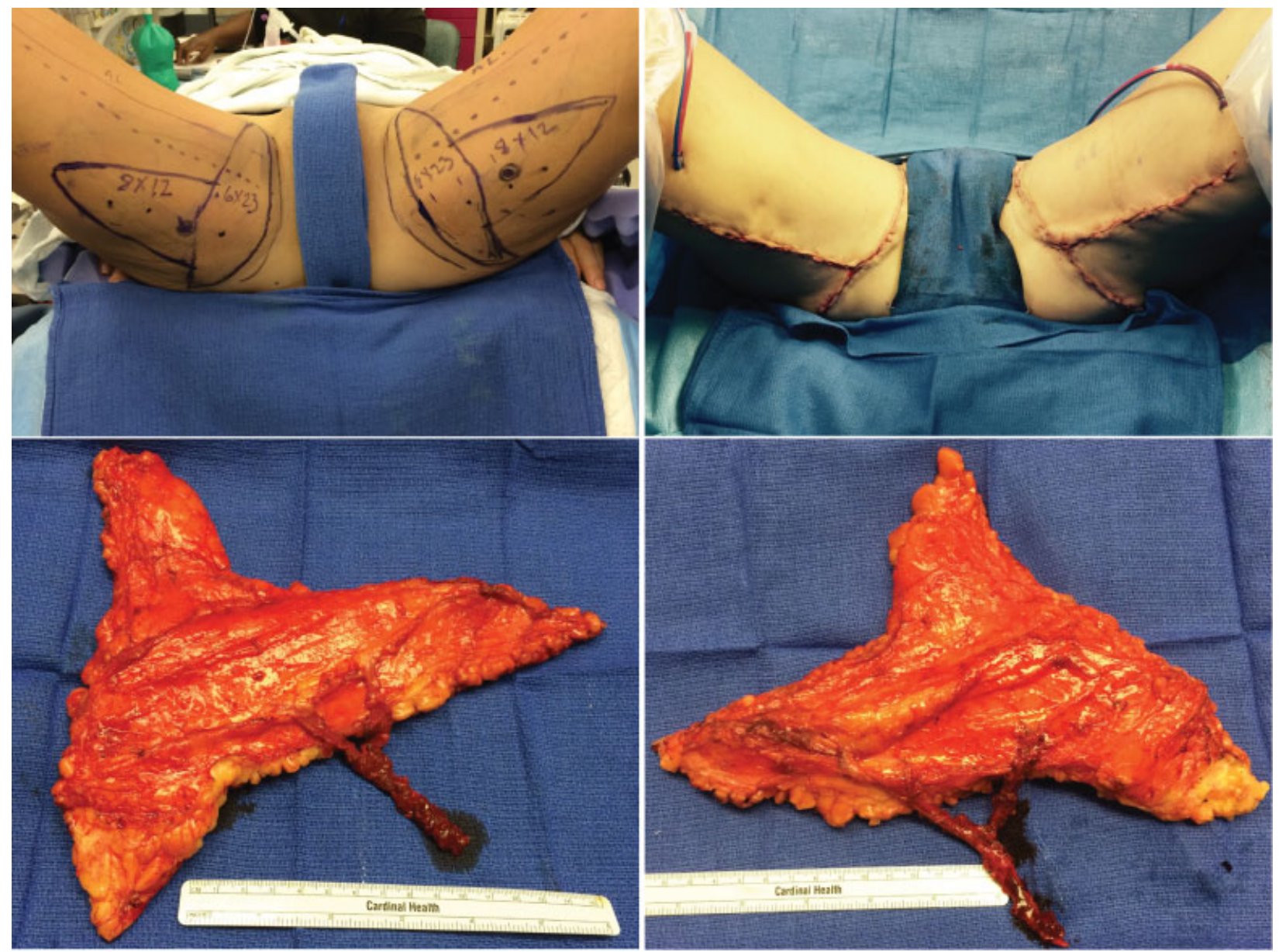

Fig. 2 Preoperative markings for the fleur-de-PAP flaps used in the bilateral breast reconstruction of our patient included two profunda artery perforators along the central axis of the vertical aspect flap, with transverse extensions of the flap, included both posteriorly and anteriorly to maximize volume (upper left). The closure of the donor site resulted in a T-shaped incision similar to an extended medial thigh lift (upper right). Each flap was raised on a single vascular pedicle, and consisted of three arms that allowed for inset in a teardrop pattern with increased projection and volume at the inferior pole once the transverse extensions were folded posteriorly to the main body of the flap (lower left and right).

\section{References}

1 Gill PS, Hunt JP, Guerra AB, et al. A 10-year retrospective review of 758 DIEP flaps for breast reconstruction. Plast Reconstr Surg 2004; 113(4):1153-1160

2 Stalder MW, Lam J, Allen RJ, Sadeghi A. Using the retrograde internal mammary system for stacked perforator flap breast reconstruction: 71 breast reconstructions in 53 consecutive patients. Plast Reconstr Surg 2016;137(2):265e-277e

3 Allen RJ, Haddock NT, Ahn CY, Sadeghi A. Breast reconstruction with the profunda artery perforator flap. Plast Reconstr Surg 2012; 129(1):16e-23e

4 Massey MF, Spiegel AJ, Levine JL, et al; Group for the Advancement of Breast Reconstruction. Perforator flaps: recent experience, current trends, and future directions based on 3974 microsurgical breast reconstructions. Plast Reconstr Surg 2009;124(3):737-751

5 Mayo JL, Allen RJ, Sadeghi A. Four- flap breast reconstruction: bilateral stacked DIEP and PAP flaps. Plast Reconstr Surg Glob Open 2015;3(5):e383. Doi: 10.1097/GOX.0000000000000353
6 Mayo JL, Canizares O, Torabi R, Allen RJS Sr, St Hilaire H. Expanding the applications of the profunda artery perforator flap. Plast Reconstr Surg 2016;137(2):663-669

7 Rutter CE, Park HS, Killelea BK, Evans SB. Growing use of mastectomy for ductal carcinoma-in situ of the breast among young women in the United States. Ann Surg Oncol 2015;22(7): 2378-2386

8 Stalder MW, Accardo K, Allen RJ, Sadeghi A. Aesthetic refinement of the abdominal donor site after autologous breast reconstruction. Plast Reconstr Surg 2015;136(3):455-461

9 Saad A, Sadeghi A, Allen RJ. The anatomic basis of the profunda femoris artery perforator flap: a new option for autologous breast reconstruction-a cadaveric and computer tomography angiogram study. J Reconstr Microsurg 2012; 28(6):381-386

10 McKane BW, Korn PT. The fleur-de-lis upper gracilis flap for breast reconstruction: flap design and outcome. Ann Plast Surg 2012; 69(4):383-386 